\title{
Análisis de variables significativas para la generación de un inventario de emisiones de fuentes móviles y su proyección
}

\author{
Analysis of significative variables for the generation of an inventory \\ of emissions from mobile sources and their projections
}

\author{
Sebastián Tolvett Caro ${ }^{1 *} \quad$ Pilar Henríquez $^{2} \quad$ Mauricio Osses $^{3}$ \\ Recibido 11 de enero de 2016, aceptado 1 de junio de 2016 \\ Received: January 11, 2016 Accepted: June 1, 2016
}

\begin{abstract}
RESUMEN
La determinación de emisiones de fuentes móviles es compleja considerando que las fuentes se desplazan por el área de interés, bajo diferentes regímenes de operación y con diversas tecnologías. El presente trabajo de investigación analiza la información disponible en instituciones públicas de Chile con el objetivo de generar un inventario de emisiones representativo, actualizable y dinámico. Respecto al crecimiento del parque, el principal indicador es la tasa de motorización, la que al 2012 superó los 207 vehículos cada mil habitantes, lejos de tasas de países desarrollados, entre 400 y 600 vehículos cada mil habitantes, por lo que la tendencia de crecimiento del parque automotriz en Chile seguirá manteniéndose. Actualmente no existe en Chile una base oficial de factores de emisión de vehículos motorizados; sin embargo, el Ministerio de Transportes y Telecomunicaciones realiza un control exhaustivo de la entrada de vehículos a través del Centro de Control y Certificación Vehicular (3CV). Este centro determina a través de mediciones directas las emisiones de cada uno de los modelos que entran al país confirmando que se cumplan los límites máximos de emisión establecidos por las normas. Como resultado de este trabajo se obtiene un inventario de emisiones de Fuentes Móviles con información local que permite proyectar emisiones, siendo un complemento a modelos tradicionales como MODEM. La proyección de emisiones al 2030 muestra una reducción de 76\% para material particulado 2,5 micrones, una reducción de 53\% en óxidos de nitrógeno y un aumento de 193\% en dióxido de carbono en relación al 2008.
\end{abstract}

Palabras clave: Emisiones, inventario, fuente, vehículo, contaminación.

\section{ABSTRACT}

Determining emissions from mobile sources is complex considering that the sources travel through the area of interest under different operating regimes and with different technologies. This research analyzes the information available in public institutions in Chile with the aim of creating an inventory of representative, upgradeable and dynamic emissions. Regarding the growth of the fleet, the main indicator is the motorization rate, which by 2012 exceeded 207 vehicles per thousand inhabitants, far from rates in developed countries, between 400 and 600 vehicles per thousand inhabitants, so the growth trend of the automotive fleet in Chile will be maintained. Currently in Chile there isn't a formal basis for emission factors from motor vehicles, however, the Ministry of Transport and Communications regulates the entry of vehicles through the Vehicle Control and Certification Center (3CV). The center determined through direct measurement emissions of each of the models entering the country confirming that the

\footnotetext{
1 Departamento de Ingeniería Mecánica. Universidad Tecnológica Metropolitana. Campus Macul, Av. José Pedro Alessandri 1242, Ñuñoa. Santiago, Chile. E-mail: stolvett@utem.cl

2 Sistemas Sustentables. Doctor Torres Boonen 731, Providencia. Santiago, Chile. E-mail: pilar@sistemas-sustentables.com

3 Departamento de Ingeniería Mecánica. Universidad Técnica Federico Santa María. Campus Santiago San Joaquín, Vicuña Mackenna 3939. Santiago, Chile. E-mail: mauricio.osses@usm.cl

* Autor de correspondencia
} 
emission standard established by the regulations is met. As a result of this work, an emissions inventory of Mobile Sources with local information for emissions projecting, as a complement to traditional models as MODEM was obtained. The projected emissions in 2030 shows a reduction of $76 \%$ for particulate matter 2.5 microns, a reduction of 53\% in nitrogen oxides and an increase of $193 \%$ carbon dioxide in relation to 2008.

Keywords: Emissions, inventory, source, vehicle, pollution.

\section{INTRODUCCIÓN}

En general, las emisiones se clasifican en emisiones provenientes de Fuentes Fijas o Estacionarias y de Fuentes Móviles. En particular, la determinación de emisiones de Fuentes Móviles es compleja debido no solo a que las fuentes se desplazan por el área de interés sino que al mismo tiempo lo hacen bajo diferentes regímenes de operación y con diversas tecnologías de motorización. El desafío de la determinación de emisiones de fuentes móviles representativas se acentúa en Chile, debido principalmente a que existen 69 marcas de vehículos livianos, 28 marcas de camiones y 18 marcas de buses, todos con sus respectivos modelos, representando más de 24 orígenes.

En cuanto a la caracterización de los tipos de vehículos que circulan en las calles del país, según el Instituto Nacional de Estadísticas (INE) el parque de vehículos para el 2013 en Chile consta de 4.168.980 vehículos motorizados, de los que $90,6 \%$ corresponden a vehículos livianos y medianos (3.781.001 unidades), 4,3\% son camiones (181.305 unidades), 2,4\% corresponden a taxis (100.505 unidades) y $1,9 \%$ buses ( 81.263 unidades). Por otro lado, las ventas de vehículos reportadas por la Asociación Nacional Automotriz de Chile (ANAC) alcanzaron las 378.240 [3] unidades en el 2013.

En relación a la brecha disponible para el crecimiento del parque, el principal indicador a observar es la tasa de motorización. El 2012 esta tasa superó los 207 vehículos cada mil habitantes [1], muy lejos de alcanzar tasas de países desarrollados las cuales están entre 400 y 600 [2] vehículos cada mil habitantes por lo que se espera que la tendencia de crecimiento del parque automotriz en Chile seguirá manteniéndose.

El Ministerio de Transportes y Telecomunicaciones realiza un control exhaustivo de la entrada de vehículos a través de su Centro de Control y Certificación Vehicular (3CV). Este centro determina, ya sea por medio de mediciones directas en el caso de vehículos livianos y medianos o de la homologación de resultados de pruebas internacionales para el caso de vehículos pesados, las emisiones de cada uno de los modelos que entran al país para confirmar que los límites máximos de emisión establecidos por la ley chilena se cumplan. Los resultados de estas pruebas y la información obtenida mediante el proceso de homologación permiten tener una base de datos muy detallada de los niveles de emisión que corresponden a cada modelo de vehículo nuevo que se integra a Chile. Esta información se puede analizar en conjunto con los antecedentes del parque vehicular existente que reporta INE cada año y con las ventas anuales que ANAC publica de manera permanente y sistemática.

En cuanto a la estimación de emisiones, empíricamente se ha observado que las mediciones en laboratorio no necesariamente representan las emisiones en ciclo real propias de una zona de estudio. Sin embargo, estas emisiones sí representan bien las tendencias tecnológicas y el efecto del origen de fabricación de los vehículos en las emisiones. Además, considerando estudios posteriores con equipos de medición portátiles (PEMS, por su sigla en inglés) u otros sistemas, es posible corregir estas emisiones según diferentes ciclos de conducción representativos de cada ciudad y, a la vez, determinar factores de deterioro de emisiones asociadas a flota vehicular en uso. En este contexto las tres fuentes de información mencionadas anteriormente $(3 \mathrm{CV}$, INE y ANAC), permiten realizar un inventario de emisiones de Fuentes Móviles con información local, lo que significa un valor agregado por sobre aquellos inventarios que se obtienen utilizando factores de emisión teóricos de fuentes internacionales de los Estados Unidos y Europa (EPA, MOVES, COPERT), especialmente para el caso de vehículos livianos y medianos que disponen de factores de emisión obtenidos por $3 \mathrm{CV}$ mediante medición directa de emisiones en el proceso de homologación. 


\section{ANÁLISIS TEÓRICO}

Existen diferentes metodologías para estimar emisiones de fuentes móviles y por consiguiente estas metodologías están agrupadas en diferentes modelos de emisiones. Sin embargo, todos los modelos utilizan dos variables importantes las que se deben definir con la mejor precisión posible.

La primera variable es la Actividad, es decir es necesario conocer los kilómetros recorridos por los vehículos a los cuales se les pretende estimar las emisiones. Es importante separar la actividad por las diferentes categorías vehiculares debido a que puede variar de gran manera dependiendo de si el vehículo tiene un uso particular, comercial o si es taxi. Existen diferentes formas de estimar la actividad, por ejemplo, utilizando el consumo de combustible Regional o Nacional, o de maneras más complejas como por ejemplo apoyándose con modelos de transporte.

La segunda variable corresponde a los Factores de Emisión, esta variable es de difícil obtención porque depende de los diferentes modelos y tipos de motorizaciones que circulan en una determinada región de estudio. Por otro lado, también depende de la forma de conducir, los niveles de congestión, la geografía, etcétera. En el caso de Chile, el Ministerio de Transportes y Telecomunicaciones mediante su laboratorio de emisiones $3 \mathrm{CV}$ registra las emisiones de cada uno de los vehículos nuevos livianos y medianos que ingresan al parque vehicular desde 1997.

Por último, se utilizan diferentes factores de corrección enfocados a corregir las variables de Actividad y Factores de Emisión. Una de las más importantes corresponde a los Factores de Deterioro, estos factores corrigen a los Factores de Emisión debido al uso de los vehículos. Existen estudios que reportan factores de deterioro medidos en Chile para el caso de vehículos livianos a gasolina [5], pero en general esta variable tiene mayores incertidumbres.

\section{Desarrollo metodológico}

En base a los antecedentes presentados anteriormente y a la disponibilidad de factores de emisión señalados previamente, se describe en la presente sección la metodología empleada en el desarrollo del modelo.
La Figura 1 resume la metodología general en la elaboración del modelo de emisiones, la que consta de tres partes principales: 1) definición de datos de entrada o inputs, 2) cálculo de emisiones, y 3) generación de reportes de emisiones.

La metodología considera cinco tipos de datos de entrada para el cálculo de emisiones vehiculares y que se resumen en Tabla 1.

\section{Calculo de emisiones}

La ecuación general que describe el cálculo de emisiones por año en el modelo se indica a continuación:

$$
E=\sum_{i j k r} F E_{i j k} * L_{i r} * N_{i j k r} * F D_{i j k}
$$

Donde:

E : emisiones totales para el parque vehicular nacional [gr/año].

$\mathrm{FE}_{i j k} \quad$ : Factor de emisión para la categoría vehicular $i$ que utiliza combustible j para el estándar de emisión $k$ [gr $/ \mathrm{km}]$.

$\mathrm{L}_{i r} \quad$ : Distancia recorrida en un año por vehículo de categoría $i$ en la región $r$ [km/veh-año]

$\mathrm{N}_{i j k r} \quad$ : Parque vehicular de la categoría $i$ que utiliza combustible $j$ para el estándar de emisión $k$ en la región $r$ [veh].

$\mathrm{FD}_{i j k}:$ Factor de deterioro para la categoría vehicular $i$ que utiliza combustible $j$ para el estándar de emisión $k$ [adimensional].

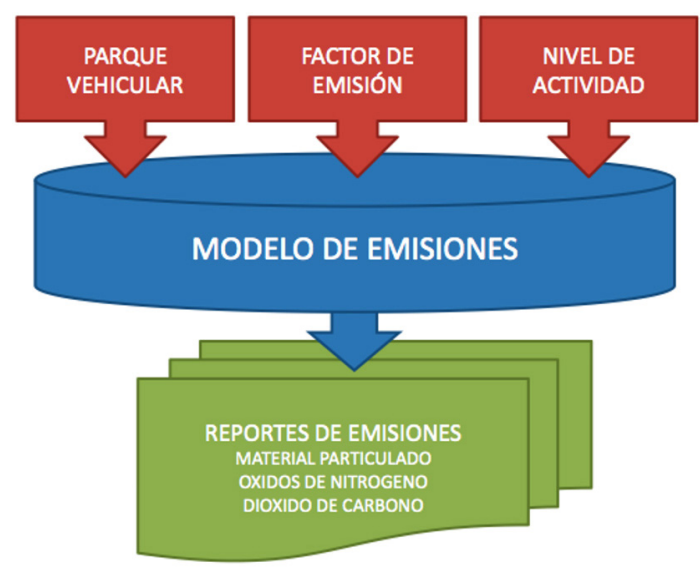

Figura 1. Diseño del Modelo de Emisiones. 
Tabla 1. Resumen de datos de entrada del modelo.

\begin{tabular}{|c|c|c|}
\hline Input & Descripción & Fuente \\
\hline Parque Vehicular & $\begin{array}{l}\text { Cantidad total de vehículos. } \\
\text { Se utilizan bases de datos del } 2008 \text { al } 2012 .\end{array}$ & Instituto Nacional de Estadísticas (INE). \\
\hline $\begin{array}{l}\text { Factores de } \\
\text { Emisión } 3 \mathrm{CV}\end{array}$ & $\begin{array}{l}\text { Tasa de emisiones por kilómetro determinadas } \\
\text { en el proceso de homologación de vehículos } \\
\text { livianos y medianos. Se consideran los } \\
\text { contaminantes NOx, MP y } \mathrm{CO}_{2} \text {. }\end{array}$ & $\begin{array}{l}\text { Centro de Control y Certificación Vehicular } \\
\text { 3CV, Ministerio de Transportes. }\end{array}$ \\
\hline $\begin{array}{l}\text { Nivel de } \\
\text { Actividad }\end{array}$ & $\begin{array}{l}\text { Corresponde al total de kilómetros que recorre } \\
\text { un vehículo de una categoría vehicular dada } \\
\text { en un año. } \\
\text { Este parámetro es definido para cada región } \\
\text { del país para una categoría vehicular dada. }\end{array}$ & $\begin{array}{l}\text { Evaluados según estudio Análisis y Desarrollo } \\
\text { de una Metodología de Estimación de Consumos } \\
\text { Energéticos y Emisiones para el Transporte } \\
\text { (SECTRA, 2010). }\end{array}$ \\
\hline $\begin{array}{l}\text { Factor de } \\
\text { Deterioro }\end{array}$ & $\begin{array}{l}\text { Corresponde al deterioro de las emisiones de } \\
\mathrm{CO}, \mathrm{HC} \text { y NOx que ocurre los vehículos a } \\
\text { gasolina con el uso. Se considera el máximo } \\
\text { de deterioro una vez que acumula } 120.000 \\
\text { kilómetros. }\end{array}$ & $\begin{array}{l}\text { Elaboración de una Estrategia Nacional } \\
\text { para Reducir la Emisión de Contaminantes } \\
\text { Atmosféricos Provenientes del Sector Transporte, } \\
\text { Ministerio de Medioambiente, } 2012 .\end{array}$ \\
\hline
\end{tabular}

Proyección de parque, actividad vehicular y obsolescencia

La estimación y proyección de emisiones tiene directa relación con tres variables explicativas: 1) proyección del parque, 2) actividad vehicular y 3) obsolescencia.

La proyección del parque vehicular esta asociada con la proyección de ventas del sector automotriz, en el presente trabajo se utilizó un modelo econométrico [6]. El modelo arroja que el parque vehicular alcanzaría los 8.352 .598 unidades al 2030 y las ventas alcanzarían las 550.000 unidades para el mismo año. En la Figura 2 se muestra la proyección del parque vehícular.

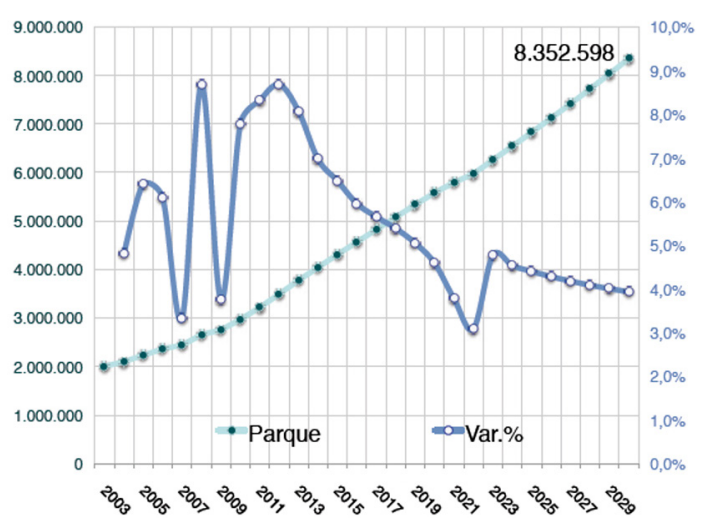

Figura 2. Proyección parque vehicular al 2030.
Trabajos anteriores muestran que la actividad vehicular, o en otras palabras, la cantidad de kilómetros que recorre un vehículo, disminuye en el tiempo alcanzando un uso mínimo en 25 años [7]. Esto tiene un impacto en las emisiones debido a que los vehículos más antiguos o más contaminantes en realidad tienen un uso menor que los nuevos. Para realizar la proyección de emisiones se requiere desarrollar la curva de obsolescencia. Esta curva indica como disminuye el parque año a año según su antigüedad.

Comparando los resultados de Actividad y Obsolescencia obtenidos de análisis de bases de datos de Plantas de Revisión Técnica (PRT) se define la Figura 3, que muestra la curva de

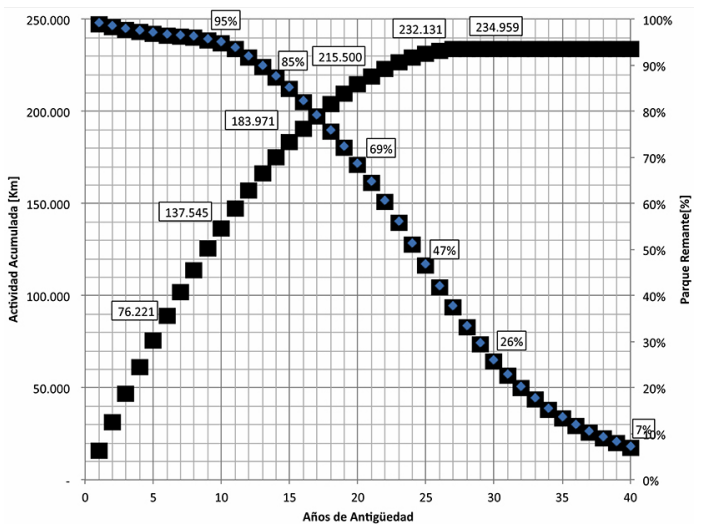

Figura 3. Actividad vehicular acumulada versus parque remanente. 
actividad acumulada y la curva de obsolescencia para un determinado año-modelo. Se observa que al décimo año de antigüedad el parque remanente es de $95 \%$ y el kilometraje acumulado alcanzaría los 137.545 kilómetros (11.073 kilómetros al año). A los 20 años aun existe un parque remanente de $69 \%$ del original con una actividad acumulada de 215.500 kilómetros (5.114 kilómetros al año) y a los 30 años sigue quedando un parque remanente de $26 \%$ con un kilometraje acumulado de 234.959 kilómetros (1.531 kilómetros al año).

\section{RESULTADOS}

A continuación se presentan los resultados de emisiones del parque total para los contaminantes $\mathrm{MP} 2,5, \mathrm{NOx}$ y $\mathrm{CO}_{2}$.

\section{Emisiones proyectadas de MP2,5}

La Figura 4 presenta la proyección al 2030 de las emisiones de MP2,5 del parque agregado. A pesar de que el parque vehicular crece de manera sostenida, las emisiones de MP2,5 al 2030 se reducen en un $77 \%$ respecto al 2008 (equivalente a 3.031 tonMP), esto producto de la entrada de las normas Euro 5 los años 2013 al 2015, lo que impacta principalmente las emisiones de MP2,5 para vehículos diésel.

Se observa además que los camiones son causantes del 79\% de las emisiones de MP2,5 en el 2030, equivalente a 709 toneladas de MP2,5. Los vehículos livianos y medianos aportarían $13 \%$ del total al 2030 (116 toneladas de MP2,5), mientras que los buses serían el 8\% restante (75 toneladas de MP2,5). El mayor impacto en las emisiones de MP2,5 en el largo plazo se produce porque, en general, los camiones se renuevan más lento que las otras dos categorías, lo que hace que hacia el 2030 exista

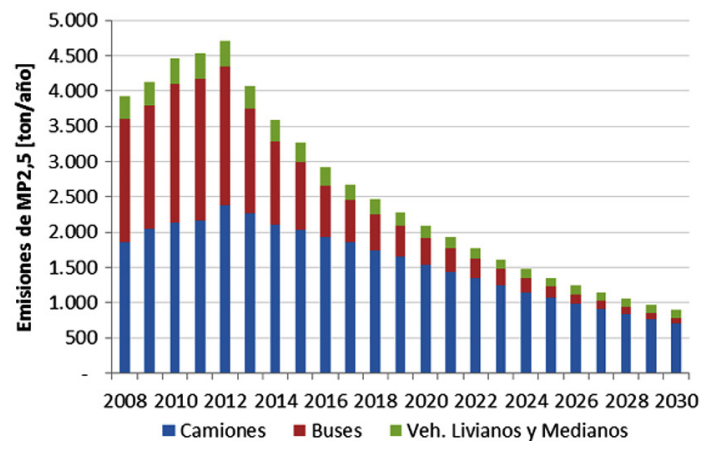

Figura 4. Proyección emisiones MP2,5 al 2030. aun una presencia relevante de camiones antiguos, $22 \%$ de la flota de camiones total en el 2030 con estándar de emisión Euro 4 o inferior.

La Tabla 2 resume los resultados de emisiones de MP2,5 para los cortes temporales 2010, 2015, 2025 y 2030.

\section{Emisiones proyectadas de NOx}

La Figura 5 presenta las emisiones de NOx proyectadas al 2030 para el parque vehicular total. Similar al caso anterior, la entrada de normas Euro 5 y Euro 6 hacen que, en general, se observe una reducción en el total de emisiones a pesar de que el parque vehicular se incremente. Luego, las emisiones de NOx disminuyen sostenidamente hacia el 2030, siendo $45 \%$ menor que las emisiones totales del 2008 y equivalente a 50.466 tonNOx. $\mathrm{La}$ reducción es menor que para el caso de emisiones de partículas, principalmente por el retraso en la norma Euro 6 (que se supuso que entraría en el 2020), estándar de emisión que impacta considerablemente

Tabla 2. Resumen emisiones MP2,5.

\begin{tabular}{|c|c|c|c|c|c|}
\hline \multicolumn{6}{|c|}{ MP2,5 (ton/año) } \\
\hline Categoría & $\begin{array}{c}\text { Sub- } \\
\text { categoría }\end{array}$ & 2010 & 2015 & 2025 & 2030 \\
\hline \multirow{2}{*}{$\begin{array}{l}\text { Liviano y } \\
\text { Mediano }\end{array}$} & Gasolina & 66 & 67 & 68 & 81 \\
\hline & Diésel & 293 & 218 & 58 & 34 \\
\hline \multirow{3}{*}{ Camiones } & Livianos & 118 & 107 & 55 & 36 \\
\hline & Medianos & 131 & 119 & 75 & 52 \\
\hline & Pesados & 1.876 & 1.805 & 937 & 621 \\
\hline \multirow{3}{*}{ Buses } & Urbano & 1.734 & 849 & 153 & 71 \\
\hline & Interurbano & 33 & 18 & 4 & 2 \\
\hline & Licitado & 204 & 89 & 2 & 2 \\
\hline \multicolumn{2}{|c|}{ Total } & 4.456 & 3.273 & 1.351 & 899 \\
\hline
\end{tabular}

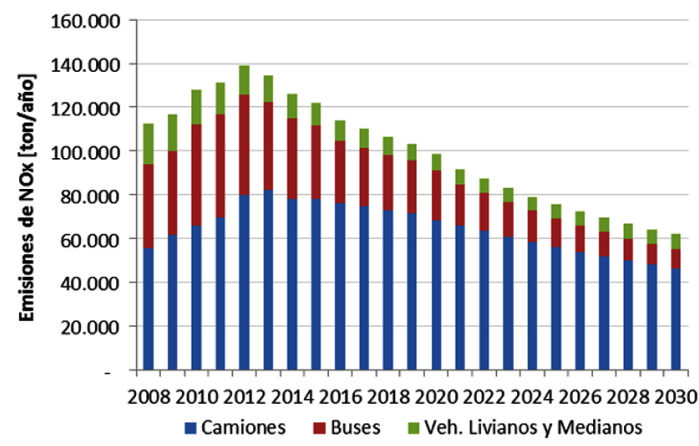

Figura 5. Proyección emisiones NOx al 2030. 
las emisiones de NOx en vehículos diésel. Por otra parte, también se observa que al 2030 los camiones emitirían el $75 \%$ de las emisiones de NOx (equivalente a 46.441 tonNOx), mientras que los buses $14 \%$ de las emisiones totales de NOx (equivalente a 8.716 tonNOx). Los vehículos livianos y medianos representarían $11 \%$ del total de emisiones de NOx en el 2030 (equivalente a 6.989 tonNOx).

La Tabla 3 resume los resultados de emisiones de NOx para los cortes temporales 2010, 2015, 2025 y 2030.

\section{Emisiones proyectadas de $\mathrm{CO}_{2}$}

La Figura 6 presenta las emisiones de $\mathrm{CO}_{2}$ proyectadas al 2030 del parque vehicular total. A diferencias de los resultados anteriores, para el caso de las emisiones de $\mathrm{CO}_{2}$ existe un incremento sostenido hacia el 2030 el cual está directamente relacionado al incremento del parque vehicular proyectado. En general, se observa que hacia el año 2030, las emisiones de $\mathrm{CO}_{2}$ aumentarán en un $193 \%$ con respecto al 2008 (equivalente a 27,7 millones de tonCO $\mathrm{C}_{2}$ ) alcanzando la cifra total de 42,2 millones de tonCO $\mathrm{C}_{2}$. Un aspecto relevante es la elevada participación en las emisiones de $\mathrm{CO}_{2}$ para los vehículos livianos y medianos, siendo en el año 2030 el $47 \%$ (equivalente a 19,9 millones de tonCO $\mathrm{CO}_{2}$ ).

Los camiones, por su parte, aumentarán sus emisiones al 2030 en 204\% respecto del 2008, llegando a un total de 15,2 millones de tonCO $\mathrm{CO}_{2}$ en el 2030. Los buses también incrementan sus emisiones al 2030 en $141 \%$ respecto al 2008 , lo que implica un

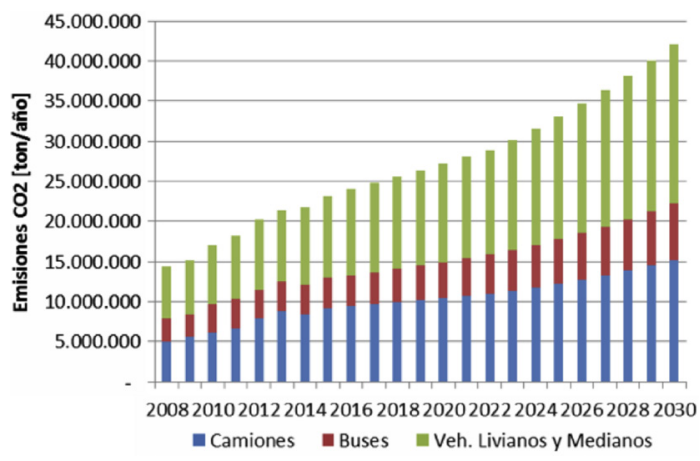

Figura 6. Proyección emisiones $\mathrm{CO}_{2}$ al 2030.

total de 7,1 millones de tonCO $\mathrm{CO}_{2}$ emitido al final del período.

La Tabla 4 resume los resultados de emisiones de $\mathrm{CO}_{2}$ para los cortes temporales 2010, 2015, 2025 y 2030.

\section{CONCLUSIONES}

Si bien la disponibilidad de diésel ultralimpio en todo el país en conjunto con la entrada de normas más exigentes Euro 5 y Euro 6 para el parque vehicular nuevo que se incorpore al país tendría un impacto positivo al 2030 en la reducción de contaminantes locales como MP2,5 y NOx, se observa que los camiones emitirían considerablemente más emisiones por unidad vehicular comparado con buses y vehículos livianos y medianos, por lo que es recomendable comenzar a evaluar alternativas de recambio tecnológico bajo distintos instrumentos que deben ser definidos considerando las limitaciones de un mercado que presenta diversos orígenes y que

Tabla 3. Resumen emisiones NOx.

\begin{tabular}{|c|c|c|c|c|c|}
\hline \multicolumn{6}{|c|}{ NOx (ton/año) } \\
\hline Categoría & Subcategoría & 2010 & 2015 & 2025 & 2030 \\
\hline \multirow{2}{*}{ Livianos } & Gasolina & 12.856 & 6.765 & 3.702 & 4.581 \\
\hline & Diésel & 2.960 & 3.569 & 2.667 & 2.408 \\
\hline \multirow{3}{*}{ Camiones } & Livianos & 3.201 & 3.463 & 2.829 & 2.765 \\
\hline & Medianos & 4.264 & 4.748 & 5.202 & 6.142 \\
\hline & Pesados & 58.564 & 69.941 & 48.008 & 37.535 \\
\hline \multirow{3}{*}{ Buses } & Urbano & 38.275 & 27.589 & 12.993 & 8.506 \\
\hline & Interurbano & 842 & 656 & 307 & 208 \\
\hline & Licitado & 7.004 & 5.148 & 2 & 2 \\
\hline \multicolumn{2}{|c|}{ Total } & 127.965 & 121.878 & 75.709 & 62.146 \\
\hline
\end{tabular}


Tabla 4. Resumen emisiones $\mathrm{CO}_{2}$.

\begin{tabular}{|c|c|c|c|c|c|}
\hline \multicolumn{6}{|c|}{$\mathrm{CO}_{2}(1.000$ ton/año $)$} \\
\hline Categoría & Subcategoría & 2010 & 2015 & 2025 & 2030 \\
\hline \multirow{2}{*}{ Livianos } & Gasolina & 5.911 & 8.000 & 12.272 & 16.137 \\
\hline & Diésel & 1.360 & 2.189 & 3.029 & 3.815 \\
\hline \multirow{3}{*}{ Camiones } & Livianos & 324 & 453 & 578 & 700 \\
\hline & Medianos & 373 & 631 & 1.205 & 1.655 \\
\hline & Pesados & 5.386 & 8.095 & 10.437 & 12.807 \\
\hline \multirow{3}{*}{ Buses } & Urbano & 2.934 & 3.114 & 4.882 & 6.296 \\
\hline & Interurbano & 73 & 83 & 136 & 175 \\
\hline & Licitado & 634 & 589 & 567 & 567 \\
\hline \multicolumn{2}{|c|}{ Total } & 16.998 & 23.155 & 33.105 & 42.152 \\
\hline
\end{tabular}

además está sujeto a diversos tratados comerciales entre países. Estas aristas añaden una complejidad adicional al momento de definir políticas públicas de recambio tecnológico.

En relación a la evolución de las emisiones asociadas a los vehículos livianos y medianos, la entrada de normas Euro 5 y Euro 6 genera reducciones considerables al 2030 de MP2,5, igualando prácticamente a los niveles de emisión actuales de los vehículos a gasolina. El análisis empírico de las tasas de emisiones medidas en el $3 \mathrm{CV}$ para la homologación de vehículos livianos y medianos Euro 5 en el país (entre el 2011 a marzo 2014), señaló que para el caso de MP2,5 las tasas de emisiones entre motorización diésel y gasolina prácticamente se igualaban, en cambio, para el caso de las tasas de NOx existía una diferencia de $85 \%$ mayor para una motorización diésel respecto a una a gasolina. Sin embargo, la entrada de una norma Euro 6 apunta a acortar esta diferencia en NOx entre ambos tipos de motorización. De lo anterior se puede inferir que, en presencia de un estándar Euro 6, ambas tecnologías para vehículos livianos y medianos (gasolina y diésel) serán similares en sus tasas de emisión por kilómetro de material particulado y óxidos de nitrógeno y no hay ventajas o desventajas importantes que respalden aplicar un tratamiento diferenciado a vehículos livianos y medianos diésel o gasolina de última generación, reiterando la condición de un estándar Euro 6 para esta categoría vehicular.

En la misma línea del análisis de la información pública disponible en las fuentes ya mencionadas, los resultados de emisiones obtenidos para el parque vehicular hacia el 2030 dejan en evidencia la relevancia de la participación de los camiones en las emisiones de los contaminantes locales MP2,5 y NOx (sobre el 70\% del total en dicho año).

Finalmente, según los escenarios evaluados para el $\mathrm{CO}_{2}$ en vehículos livianos, desincentivar la motorización diésel hoy puede producir el efecto contrario a los acuerdos internacionales adquiridos por el país que buscan la reducción de emisiones de gases de efecto invernadero. En este sentido, es relevante considerar que el $47 \%$ de las emisiones de $\mathrm{CO}_{2}$ al 2030 son generadas por el parque de vehículos livianos y medianos, por lo que se recomienda comenzar a evaluar medidas que apunten a que este segmento sea menos carbono intensivo por kilómetro recorrido. El fomento a nuevas tecnologías como híbridas o eléctricas puede representar una alternativa real de mitigación de emisiones de $\mathrm{CO}_{2}$, $\mathrm{u}$ otras observadas a nivel internacional, como la limitación de emisiones de $\mathrm{CO}_{2}$ para el promedio del parque de vehículos livianos que año a año se venden en países de Europa y otros países como Estados Unidos, Japón, México, entre otros.

\section{AGRADECIMIENTOS}

Agradecimientos a la Asociación Nacional Automotriz de Chile (ANAC) por el aporte de información y financiamiento del trabajo investigativo.

\section{REFERENCIAS}

[1] ANAC. "Anuario ANAC". 2012.

[2] CEI, FMI, Eurostat. "Base de Datos en Línea de Estadística de Transporte 
de América del Norte". Ministerio de Transporte de Japón, Instituto Nacional de Estadísticas de Chile, Asociación de Fábricas de Automotores de Argentina (ADEFA), Australian Bureau of Statistic.

[3] M. Osses y A. Henríquez. "Modelo de Emisiones Vehiculares MODEM". XI Congreso Chileno de Ingeniería de Transporte. 2003.

[4] ANAC. "Anuario ANAC". 2015.

[5] R. Corvalán y D. Vargas. "Experimental determination of emission deterioration factors for light duty catalytic vehicles". Transportation Research Part D: Transport and Environment. Vol. 8, Issue 4, July 2003. ISSN: 1361-9209. URL: http://www. sciencedirect.com/science/journal/13619209

[6] J.F. Cáceres y R. Díaz. "Proyecciones de Ventas de Automóviles". Inteligencia de Negocios. 2013.

[7] J. Lents y M. Osses. "Santiago Activity Study". University of California Riverside. 2001. 